\title{
Equalization for the dummy-head-headphone system capable of reproducing true directional information
}

\author{
Kimitoshi Fukudome \\ Department of Acoustic Design, Kyushu Institute of Design, \\ 226 Shiobaru, Minami-ku, Fukuoka, 815 Japan
}

(Received 18 June 1979)

\begin{abstract}
Of the dummy-head-headphone system, consisting of a dummy-head, headphones, and a listener, a method of equalization for precise reproduction of the original sound is studied. Three items are examined; (1) To express sound pressure at the eardrum quantitatively by taking account of the influence of the earcanal being closed by the headphone, (2) To obtain required characteristics of the equalizer, (3) To make clear the condition to be satisfied in order that the characteristics of the equalizer are independent of the direction of incident sounds. As a result, the required characteristics of the equalizer are given in terms of whole elements of the system. And it is found that the external shape of the dummy-head should be identical to the shape of the head of the listener in order that the equalizer suffices the requirements irrespective of the direction of incident sounds. The validity of the equalizer is verified by the experiment using the spherical head.
\end{abstract}

PACS number: 43. 88. Md, 43. 88. Vk

\section{INTRODUCTION}

The dummy-head-headphone system through which a listener is able to hear the same sound as he would hear in the sound field where the dummyhead is located may play an important role in some acoustic measurements, e.g., in evaluation of acoustics of a room or in evaluation of the acoustical devices for the reproduction of sound.

In order to make the system have the function above mentioned, it should be necessary to provide each channel of the system with an equalizer of appropriate characteristics. And it is a prerequisite for the equalizer to suffice the function without any change in its characteristics irrespective of the direction of incident sounds.

The purpose of this paper is to discuss how to realize the equalizer in terms of physical characteristics of whole elements of the system.

In the analysis, the acoustic system of human ear is divided into two parts at the entrance of the earcanal. And an equivalent circuit of the listener's ear in the sound field and that of the dummy-headheadphone system are derived. Being equated the sound pressure at the eardrum of one of the listener's ears in the sound field to the sound pressure at the same eardrum excited by the headphone connected to the corresponding microphone of the dummyhead in the sound field through the equalizer, the required characteristics of the equalizer are obtained in terms of the elements of the equivalent circuits.

The method of measuring these elements is given. Finally, the validity of the equalizer thus obtained is verified by the experiment on the spherical model head.

\section{EQUIVALENT CIRCUIT REPRESEN- TATION OF THE REAL EAR}

Any human acoustic element shows an individual difference. The head, torso, and auricle are very difficult to specify their shapes in simple terms and also to describe their acoustic properties. At audio 
frequencies, on the other hand, the earcanal and eardrum can be regarded as a one-dimensional uniform transmission line terminated in an eardrum impedance, since the cut-off frequency of the first transverse mode excited in cylindrical earcanal of $0.75 \mathrm{~cm}$ in diameter is about $27 \mathrm{kHz}$. The acoustic system of human ear can therefore be divided into two parts at the entrance of earcanal.

\subsection{Real Ear of a Listener in a Sound Field}

Consider the transmission of sounds from sound sources to one ear of a listener in the sound field. The acoustic system composed of sound sources and the listener is shown in Fig. 1. $Z_{\mathrm{T}}$ is the eardrum impedance, $l$ the length of the earcanal, and $x$ the distance along the axis of the earcanal. The head, torso, and auricle are included in the spatial acoustic network. Being referred to the output terminals of the spatial acoustic network, let the network be replaced by its Thévenin equivalent. The result is shown in Fig. 2. $P_{\mathrm{s}}(x=0)$ and $Z_{\mathrm{s}}(x=0)$ are the Thévenin pressure and the Thévenin acoustic impedance at the earcanal entrance, respectively. $P(x=x)$ and $U(x=x)$ are the sound pressure and the volume velocity at $x=x$, respectively. Note that the Thévenin acoustic impedance corresponds to the acoustic radiation impedance of a virtual plane piston without mass at the entrance of the earcanal.

The equations of the network in Fig. 2 can be written in matrix form as

$$
\left[\begin{array}{c}
P_{\mathrm{s}}(x=0) \\
U(x=0)
\end{array}\right]=K_{\mathrm{s}}(x=0) F_{l}\left[\begin{array}{l}
P(x=l) \\
U(x=l)
\end{array}\right],
$$

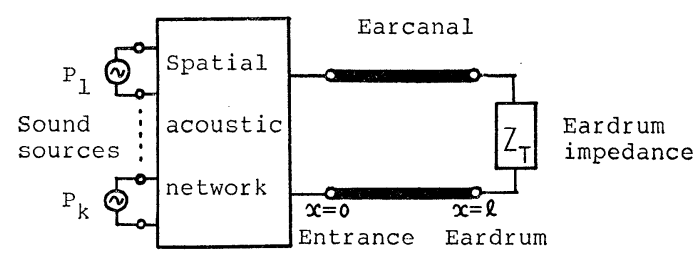

Fig. 1 Acoustic system representing the listener in the original sound field.

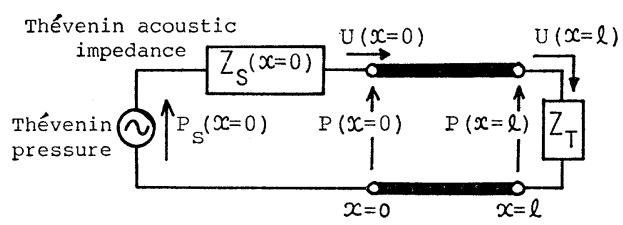

Fig. 2 Equivalent circuit of the system shown in Fig. 1. where

$$
K_{\mathrm{s}}(x=0)=\left[\begin{array}{cc}
1 & Z_{\mathrm{s}}(x=0) \\
0 & 1
\end{array}\right],
$$

and $F_{l}$ is the chain matrix of the acoustic transmission line of length $l$;

$$
F_{l}=\left[\begin{array}{cc}
\cosh \gamma l & Z_{\mathrm{c}} \sinh \gamma l \\
\left(1 / Z_{\mathrm{c}}\right) \sinh \gamma l & \cosh \gamma l
\end{array}\right],
$$

and where $\gamma$ and $Z_{\mathrm{c}}$ are the propagation constant and the characteristic impedance of the transmission line respectively. (In lossless case, $\gamma=j \omega / c=j k$ and $Z_{\mathrm{c}}=\rho c / S$, where $\rho$ is air density, $c$ sound velocity, and $S$ tube area. The hyperbolic functions then reduce to circular functions which are purely reactive.)

If the acoustic system is divided into two parts at the point $x=m$,

$$
\left[\begin{array}{l}
P_{\mathrm{s}}(x=m) \\
U(x=m)
\end{array}\right]=K_{\mathrm{s}}(x=m) F_{l-m}\left[\begin{array}{l}
P(x=l) \\
U(x=l)
\end{array}\right],
$$

where

$$
K_{\mathrm{s}}(x=m)=\left[\begin{array}{cc}
1 & Z_{\mathrm{s}}(x=m) \\
0 & 1
\end{array}\right],
$$

and $F_{l-m}$ is the chain matrix of the transmission line of length $l-m$.

Referring to Fig. 3, we obtain

$$
\begin{aligned}
P_{\mathrm{s}}(x=m)= & P_{\mathrm{s}}(x=0) /[\cosh \gamma m \\
& \left.+\left(Z_{\mathrm{s}}(x=0) / Z_{\mathrm{c}}\right) \sinh \gamma m\right], \\
Z_{\mathrm{s}}(x=m)= & Z_{\mathrm{c}} \frac{Z_{\mathrm{s}}(x=0)+Z_{\mathrm{c}} \tanh \gamma m}{Z_{\mathrm{c}}+Z_{\mathrm{s}}(x=0) \tanh \gamma m} .
\end{aligned}
$$

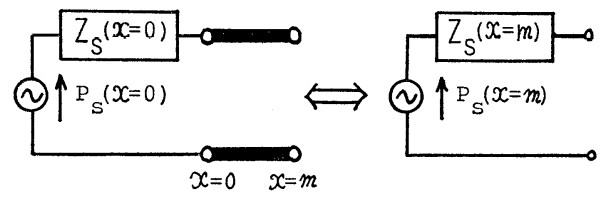

Fig. 3 Further equivalent circuit of the system left side of the point $x=m$.

\subsection{Real Ear with a Headphone}

Figure 4 shows the external ear with a headphone. Since the auricle is pressed against the temple, the concha and the neighborhood of the earcanal entrance are deformed. Besides, there are differences between individuals about the shape of auricle. 


\section{K. FUKUDOME: EQUALIZATION FOR THE DUMMY-HEAD-HEADPHONE SYSTEM}

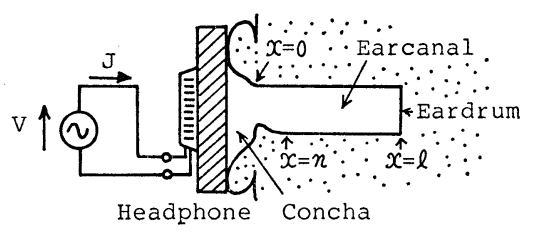

Fig. 4 External ear with the headphone.

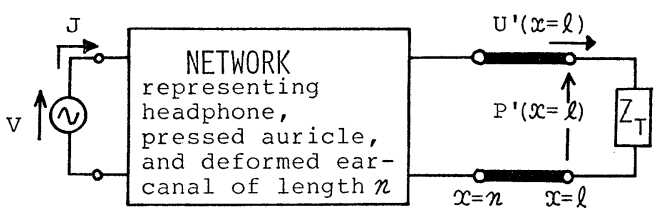

Fig. 5 Equivalent circuit of the system shown in Fig. 4.

In order to describe quantitatively the sound pressure at the eardrum generated by the headphone when coupled to a specific ear, the part from the electrical terminals of the headphone to the deformed earcanal can therefore be regarded as an electroacoustic two-port network. Figure 5 shows an equivalent circuit of the system in Fig. 4. Here the earcanal is deformed up to $x=n$.

Let $H(x=n)$ denote the chain matrix of the twoport network. The equations of the network in Fig. 5 can be written in matrix form as

$$
\left[\begin{array}{l}
V \\
J
\end{array}\right]=H(x=n) F_{l-n}\left[\begin{array}{l}
P^{\prime}(x=l) \\
U^{\prime}(x=l)
\end{array}\right],
$$

where $V$ and $J$ are the input voltage and current of the headphone respectively, $P^{\prime}(x=l)$ and $U^{\prime}(x=l)$ the sound pressure and volume velocity at the eardrum respectively, and $F_{l-n}$ the chain matrix of the undeformed earcanal of length $l-n$.

\section{EQUALIZATION FOR THE DUMMY-HEAD-HEADPHONE SYSTEM}

\subsection{Dummy-head-headphone System}

The block diagram of the dummy-head-headphone system is shown in Fig. 6. The equalizer is inserted in each channel of the system through which the listener is able to hear the same sound that the listener would hear in the original sound field.

\subsection{Representation of the Equalizer}

The equalizer is represented as shown in Fig. 7, by means of a voltage-controlled voltage source with the voltage transfer ratio $B$.

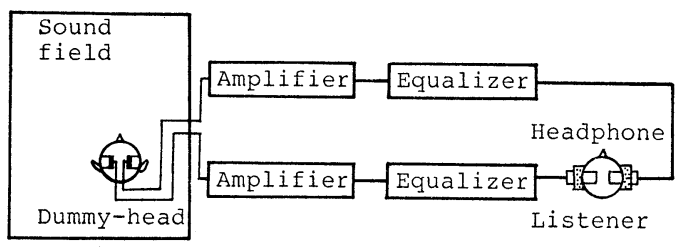

Fig. 6 Block diagram of the dummy-headheadphone system.

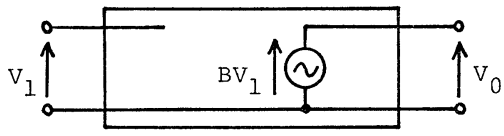

Fig. 7 Functional representation of the equalizer.

3.3 Equivalent Circuit of the Dummy-head-headphone System

Let the microphone be set in the earcanal of the dummy-head at $x=m$. Similar to the previoussection, the equivalent circuit of the dummy-headheadphone system can be shown as in Fig. 8. Here, $P_{\mathrm{sd}}(x=m)$ and $Z_{\mathrm{sd}}(x=m)$ are the Thévenin pressure and Thévenin acoustic impedance at the position $x=m$ in the earcanal of the dummy-head respectively. $A$ is the transfer ratio associated with the microphone-amplifier combination, which is the output voltage divided by the input sound pressure. $Z_{\mathrm{M}}$ is the terminating impedance of the earcanal of the dummy-head. $P_{\mathrm{d}}(x=m)$ is the sound pressure picked up by the dummy-head microphone. $B(x=m)$ denotes the voltage transfer ratio of the equalizer, when the pick-up point is at $m$.

Network equations for Fig. 8 are written as

$$
\begin{gathered}
P_{\mathrm{d}}(x=m)=P_{\mathrm{sd}}(x=m) Z_{\mathrm{M}} /\left(Z_{\mathrm{sd}}(x=m)+Z_{\mathrm{M}}\right), \\
V=B(x=m) \cdot A \cdot P_{\mathrm{d}}(x=m), \\
{\left[\begin{array}{c}
V \\
J
\end{array}\right]=H(x=n) F_{l-n}\left[\begin{array}{c}
P^{\prime}(x=l) \\
U^{\prime}(x=l)
\end{array}\right] .}
\end{gathered}
$$

Rearranging these equations, we obtain

$$
\begin{array}{r}
{\left[\begin{array}{c}
B(x=m) \cdot A \cdot Z_{\mathrm{M}} \cdot P_{\mathrm{sd}}(x=m) \\
Z_{\mathrm{sd}}(x=m)+Z_{M}
\end{array}\right]} \\
J \\
=H(x=n) F_{l-n}\left[\begin{array}{l}
P^{\prime}(x=l) \\
U^{\prime}(x=l)
\end{array}\right] .
\end{array}
$$




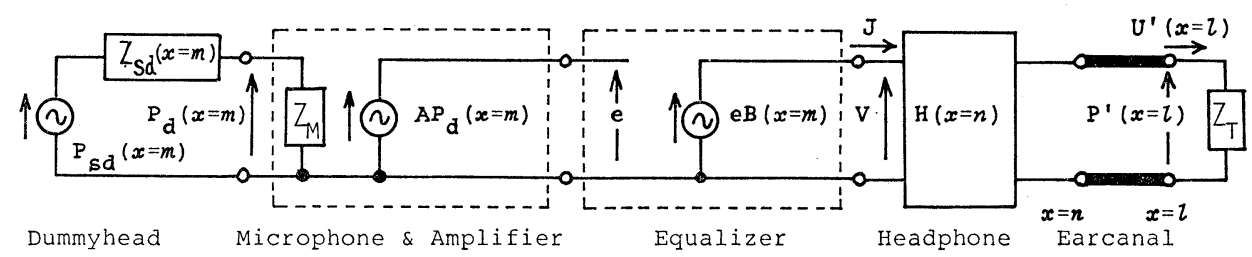

Fig. 8 Equivalent circuit of the dummy-head-headphone system.

3.4 Required Characteristics of the Equalizer, and the Necessary Condition for the Required Characteristics Being Constant Irrespective of the Direction of Incident Sounds

Let the Thévenin pressure ratio $C_{\mathrm{ps}}(x=m)$ at the position $x=m$ in the earcanal of the listener and the dummy-head be

$$
C_{\mathrm{ps}}(x=m)=P_{\mathrm{s}}(x=m) / P_{\mathrm{sd}}(x=m) .
$$

Equating $P(x=l)$ of Eq. (4) to $P^{\prime}(x=l)$ of Eq. (11) by making use of Eq. (12), we obtain the required characteristics, i.e., the voltage transfer ratio of the equalizer $B(x=m)$ as follows,

$$
\begin{aligned}
B(x=m)= & \frac{1}{A} \frac{S_{11}+\left(S_{12} / Z_{\mathrm{T}}\right)}{T_{11}+\left(T_{12} / Z_{\mathrm{T}}\right)} \\
& \cdot\left[1+\frac{Z_{\mathrm{sa}}(x=m)}{Z_{\mathrm{M}}}\right] C_{\mathrm{ps}}(x=m),
\end{aligned}
$$

where

$$
\left.\begin{array}{l}
S_{11}=h_{11} \cosh \gamma(l-n)+\left(h_{12} / Z_{\mathrm{c}}\right) \sinh \gamma(l-n) \\
S_{12}=h_{11} Z_{\mathrm{c}} \sinh \gamma(l-n)+h_{12} \cosh \gamma(l-n) \\
T_{11}=\cosh \gamma(l-m)+\left[Z_{\mathrm{s}}(x=m) / Z_{\mathrm{c}}\right] \sinh \gamma(l-m) \\
T_{12}=Z_{\mathrm{c}} \sinh \gamma(l-m)+Z_{\mathrm{s}}(x=m) \cosh \gamma(l-m)
\end{array}\right\}
$$

and $h_{11}$ and $h_{12}$ are $(1,1)$ element and $(1,2)$ element of the chain matrix $H(x=n)$, respectively.

Thus, $B(x=m)$ is expressed in terms of elements of the preceding equivalent circuits.

Let us examine the necessary condition for the characteristics of the equalizer being constant for a specific listener irrespective of the direction of incident sounds. It is enough for this purpose to examine whether the Thévenin acoustic impedance and the Thévenin pressure ratio depend on the direction of incident sounds, since other elements in the expression for $B(x=m)$ do not depend on the direction of incident sounds. As previously mentioned, the Thévenin acoustic impedance corresponds to the acoustic radiation impedance of the virtual piston in the earcanal. When the reaction of reflecting surfaces around the listener on the virtual piston may be neglected, the acoustic radiation impedance of the virtual piston depends on the shape of the head and does not depend on the location of sound sources. Thus, the Thévenin acoustic impedance $Z_{\mathrm{s}}$ is uniquely determined for a specific listener.

On the other hand, the Thévenin pressure $P_{\mathrm{s}}$ $(x=m)$ corresponds to the sound pressure generated at $x=m$ when the earcanal is blocked by a rigid plug at $x=m$. And the amplitude and phase of $\boldsymbol{P}_{\mathrm{s}}$ $(x=m)$ depends on the direction of incident sounds. When the shape of the dummy-head is not identical to that of the listener's head, the Thévenin pressure ratio $C_{\mathrm{ps}}(x=m)$ for the listener and the dummy-head would therefore be dependent on the direction of incident sounds.

Therefore, the necessary condition for the characteristics of the equalizer being constant for a specific listener irrespective of the direction of incident sounds is satisfied if the shape of the dummy-head is identical to that of the listener's head except its bottom section of earcanal where the microphone is mounted.

Then, $C_{\mathrm{ps}}(x=m)=1$, and $Z_{\mathrm{s}}(x=m)=Z_{\mathrm{sd}}(x=m)$, and the characteristics of the equalizer become

$$
B(x=m)=\frac{1}{A} \frac{S_{11}+\left(S_{12} / Z_{\mathrm{T}}\right)}{T_{11}+\left(T_{12} / Z_{\mathrm{T}}\right)}\left[1+\frac{Z_{\mathrm{s}}(x=m)}{Z_{\mathrm{M}}}\right] .
$$

Note that $B$ is always influenced by $Z_{\mathrm{s}}$ except for the use of the following dummy-head: The shape of the dummy-head is identical to that of the listener's head, the earcanal of the dummy-head is terminated with the microphone at the position corresponding to the listener's eardrum position, and the terminating impedance is equal to the listener's eardrum impedance. For this case,

$$
m=l, Z_{\mathrm{M}}=Z_{\mathrm{T}}, C_{\mathrm{ps}}(x=l)=1, Z_{\mathrm{s}}(x=l)=Z_{\mathrm{sd}}(x=l),
$$

and then, 


\section{K. FUKUDOME: EQUALIZATION FOR THE DUMMY-HEAD-HEADPHONE SYSTEM}

$$
\begin{aligned}
B(x=l) & =\frac{1}{A} \frac{S_{11}+\left(S_{12} / Z_{\mathrm{T}}\right)}{1+\left[Z_{\mathrm{s}}(x=l) / Z_{\mathrm{T}}\right]}\left[1+\left(Z_{\mathrm{s}}(x=l) / Z_{\mathrm{M}}\right)\right] \\
& =\frac{1}{A}\left[S_{11}+\left(S_{12} / Z_{\mathrm{T}}\right)\right] .
\end{aligned}
$$

Also, Eq. (15) can be used to evaluate the influences of the length of the earcanal and the eardrum impedance on the equalizer when sound is picked up at a different position in the earcanal of the dummy-head.

\section{METHOD OF MEASURING $Z_{s}, \boldsymbol{P}_{s}$, AND $\boldsymbol{H}$}

The characteristics of the equalizer have been expressed in terms of elements of the preceding equivalent circuits. These elements may be determined by measurements. Methods of measuring the eardrum impedance and earcanal dimensions are dealt in the literature. ${ }^{1)}$

Here, methods of measuring $Z_{\mathrm{s}}, P_{\mathrm{s}}$, and $H$ are described. The principle of these methods, similar to "the two-load method" by Egolf et al.,2) is as follows: When two known impedances are successively connected at the acoustic terminals, the complex pressure drops across the impedance are measured. Then we can calculate the unknown values from the measured values.

\subsection{Method of Measuring $Z_{\mathrm{s}}$ and $P_{\mathrm{s}}$}

When two known impedances $Z_{1}$ and $Z_{2}$ at aa' in Fig. 9 are connected successively, $Z_{\mathrm{s}}$ and $P_{\mathrm{s}}$ are obtained as follows:

$$
\begin{gathered}
Z_{\mathrm{s}}=\frac{\left(P_{2} / P_{1}\right)-1}{\left(1 / Z_{1}\right)-\left(P_{2} / P_{1}\right)\left(1 / Z_{2}\right)}, \\
P_{\mathrm{s}}=\frac{Z_{1}-Z_{2}}{\left(Z_{1} / P_{1}\right)-\left(Z_{2} / P_{2}\right)},
\end{gathered}
$$

where $P_{1}$ and $P_{2}$ are the complex pressure drops across the impedance $Z_{1}$ and $Z_{2}$, respectively.

Since the acoustic terminal $\mathrm{aa}^{\prime}$ corresponds to the entrance plane of the earcanal in measuring $P_{\mathrm{s}}(x=0)$ and $Z_{\mathrm{s}}(x=0)$, the impedance $Z$ is the input impedance of the earcanal measured inward. In the earcanal of the listener, it is difficult to obtain such

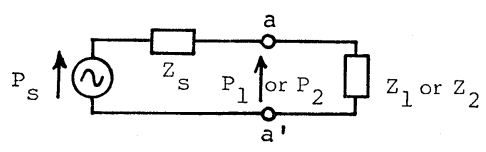

Fig. 9 Thévenin's equivalent circuit terminated in a known impedance $Z$. two known impedances.

In the case of the dummy-head, which is identical to the listener's head except the cylindrical earcanal length of which is variable and which is terminated in a known impedance $Z_{\mathrm{M}}$, such two known impedances are realizable. For the length $l, Z$ is calculated from the following equation;

$$
Z=\frac{\cosh \gamma l+\left(Z_{\mathrm{c}} / Z_{\mathrm{M}}\right) \sinh \gamma l}{\left(1 / Z_{\mathrm{c}}\right) \sinh \gamma l+\left(1 / Z_{\mathrm{M}}\right) \cosh \gamma l} .
$$

Therefore, if two lengths of the earcanal $l_{1}$ and $l_{2}$ are successively set in the dummy-head placed in the original sound field, $Z_{\mathrm{s}}$ and $\boldsymbol{P}_{\mathrm{s}}$ are calculated from Eqs. (16) and (17), respectively.

Particular care must be taken that there occurs no change at the location of the dummy-head when adjusting the length of the earcanal of the dummyhead.

\subsection{Method of Measuring $H$}

By the definition of the chain matrix, with reference of Fig. 10,

$$
\left[\begin{array}{l}
V \\
J
\end{array}\right]=H\left[\begin{array}{l}
P \\
U
\end{array}\right]=\left[\begin{array}{ll}
h_{11} & h_{12} \\
h_{21} & h_{22}
\end{array}\right]\left[\begin{array}{l}
P \\
U
\end{array}\right] .
$$

Let us obtain $H$ from the value of $V, J, P$, and $U$. Since it is difficult to make a measurement of volume velocity $U$, let us connect a known impedance $Z$ at $\mathrm{aa}^{\prime}$ and use the relation $U=P / Z$.

If two known impedances $Z_{1}$ and $Z_{2}$ are successively connected at $\mathrm{aa}^{\prime}$, we obtain

$$
\left.\begin{array}{rl}
h_{11}= & \frac{\left(V_{1} / P_{1}\right)\left(1 / Z_{2}\right)-\left(V_{2} / P_{2}\right)\left(1 / Z_{1}\right)}{\left(1 / Z_{2}\right)-\left(1 / Z_{1}\right)} \\
h_{12} & =\frac{\left(V_{2} / P_{2}\right)-\left(V_{1} / P_{1}\right)}{\left(1 / Z_{2}\right)-\left(1 / Z_{1}\right)} \\
h_{21} & =\frac{\left(J_{1} / P_{1}\right)\left(1 / Z_{2}\right)-\left(J_{2} / P_{2}\right)\left(1 / Z_{1}\right)}{\left(1 / Z_{2}\right)-\left(1 / Z_{1}\right)} \\
h_{22} & =\frac{\left(J_{2} / P_{2}\right)-\left(J_{1} / P_{1}\right)}{\left(1 / Z_{2}\right)-\left(1 / Z_{1}\right)}
\end{array}\right\}
$$

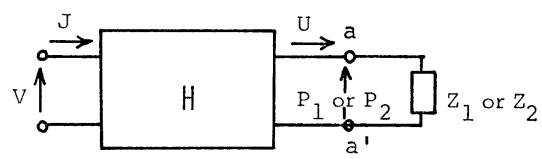

Fig. 10 Electroacoustic two-port network. The acoustic terminal is loaded by a known impedance $Z$. 
where the subscripts of $V, J$, and $P$ correspond to the subscripts of the termination $Z$.

The acoustic terminal aa' corresponds to the plane $x=n$ in the earcanal. Similar to Section 4.1, the dummy-head can be used to realize the two known impedances, $Z_{1}$ and $Z_{2}$. The external form of the dummy-head is identical to that of the listener's head with the headphone except the cylindrical earcanal (which is attached at $x=n$ ) length of which is variable and which is terminated in a known impedance $Z_{\mathrm{M}}$.

Particular care must be taken that there occurs no change in all attaching conditions of the headphone when adjusting the length of the earcanal of the dummy-head.

\section{MODEL EXPERIMENT}

In the experimental study for verifying the validity of the present method, we can adopt a spherical head as a model of the listener's head without losing the generality. Two spherical dummy-heads were used. One was the dummy-head "DH1" consisting of a spherical shell, the earcanal of length $l_{1}\left(l_{1}=\right.$ $1.00 \mathrm{~cm}$ ) terminated with a microphone, and the neck with supporting stand, as shown in Fig. 11. The other was the dummy-head "DH2" consisting of the same spherical shell as DH1, the earcanal of length $l_{2}\left(l_{2}=2.25 \mathrm{~cm}\right)$, and the neck.

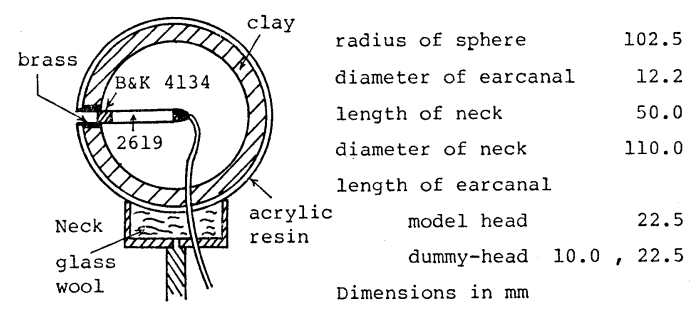

Fig. 11 Cross-sectional sketch of the spherical head.

Here, the two known impedances were obtained by the two dummy-heads being replaced. With accurate and easy setting of the microphone, the measurement of the sound pressure in the earcanal was made by the terminating microphone of the earcanal, not by the probe-microphone. The experiments were made in an anechoic chamber.

5.1 Measurement of the Thévenin Acoustic Impedance $Z_{\text {s }}$

As mentioned in Section 4.1, two dummy-heads

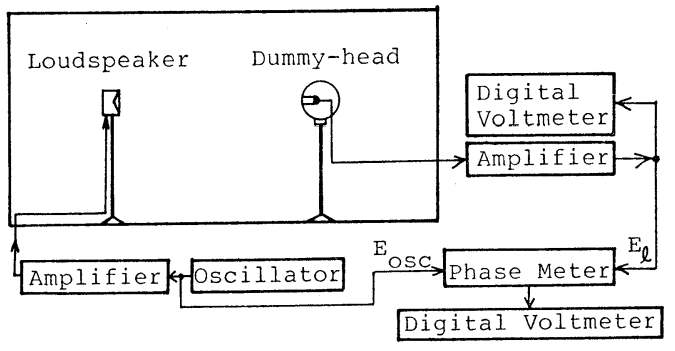

Fig. 12 Experimental arrangement for the measurement of $Z_{\mathrm{s}}$.

with the earcanal of length $l_{1}$ and $l_{2}$ were successively placed in the sound field. The sound pressure $P$ at the entrance and the sound pressure $P_{l}$ at the termination of the earcanal are in the following relation:

$$
P=\left[\cosh \gamma l+\left(Z_{\mathrm{c}} / Z_{\mathrm{M}}\right) \sinh \gamma l\right] P_{l} .
$$

By use of the measured values of $P_{l 1}$ and $P_{l 2}, Z_{\mathrm{s}}$ is obtained from Eqs. (16), (18) and (21). Figure 12 shows a block diagram of measuring procedure of $Z_{\mathrm{s}}$. Here the following relation is used:

$$
\frac{P_{l 2}}{P_{l 1}}=\frac{E_{l 2}}{E_{l 1}}=\frac{\left|E_{l 2}\right|}{\left|E_{l 1}\right|} / \arg \frac{E_{l 2}}{E_{\text {ose }}}-\arg \frac{E_{l 1}}{E_{\text {osc }}} .
$$

The sound sources were placed at a distance $1.54 \mathrm{~m}$ from the dummy-head in the horizontal plane where the central axis of the earcanal lay. The measurements were made at four different azimuthal angles of incidence, $\theta=0^{\circ}, 30^{\circ}, 60^{\circ}$, and $90^{\circ}$. The loud-

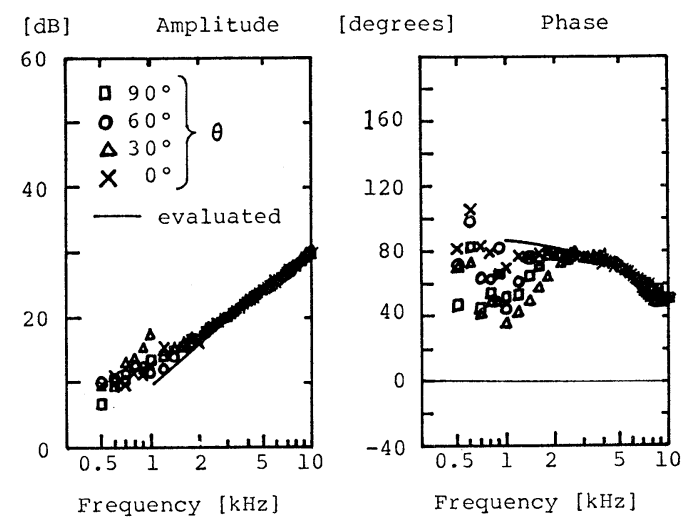

Fig. 13 Thévenin acoustic impedance at the entrance of the earcanal of the spherical head, measured at four azimuthal angles of incidence.

The zero level in the amplitude characteristics is taken as 1 cgs acoustic ohm. 


\section{K. FUKUDOME: EQUALIZATION FOR THE DUMMY-HEAD-HEADPHONE SYSTEM}

speaker used as the source was $9 \mathrm{~cm}$ full-range single cone loudspeaker with the enclosure of dimensions $103 \mathrm{~mm}(\mathrm{~W}) \times 181 \mathrm{~mm}(\mathrm{H}) \times 127 \mathrm{~mm}(\mathrm{D})$.

Figure 13 shows the obtained $Z_{\mathrm{s}}(x=0)$. It can be seen that $Z_{\mathrm{s}}$ does not depend on the angle of incident sound. The deviations at low frequencies are considered to be ascribed to the instrumental errors, with reference to Eq. (16), since $Z_{1} \gg Z_{\mathrm{s}}$, $Z_{2} \gg Z_{\mathrm{s}}$ and $P_{1}$ is therefore comparable with $P_{2}$ at those frequencies. The solid line in Fig. 13 indicates the computed value of the acoustic radiation impedance of a piston set in a sphere of the same radius as used here. Although the dummy-head used differs from the entire sphere, the result obtained shows a good agreement with the evaluated value. It suggests that $Z_{\mathrm{s}}$ obtained is reasonable.

\subsection{Measurement of the Chain Matrix $H$}

Figure 14 shows a block diagram of measuring procedure of $H$. Here, the sound pressure $P(x=x)$ is obtained from the relation $P(x=x)=E(x=x) / A$. The measurement of $A$ was separately made by means of the electrostatic actuator provided with the $\mathrm{B} \& \mathrm{~K}$ 4142 microphone calibration apparatus. The headphone used was Sennheiser HD414X (impedance $2 \mathrm{k} \Omega$ ).

Figure 15 shows the obtained chain matrix $H$. Note that $h_{21}$ and $h_{22}$ are plotted by being multiplied by the factor $10^{4}$ and 10 in the amplitude characteristics, respectively.

\subsection{Calculation of the Equalizer Characteristics}

The dummy-head-headphone system considered in this experimental study is composed of the dummy-head DH1, the headphone, and the listener who has the same head as DH2 and who has the eardrum impedance $Z_{\mathrm{T}}$ which is equal to the terminating impedance $Z_{\mathrm{M}}$ of the earcanal of $\mathrm{DH} 2$. Hence, $m=1.0[\mathrm{~cm}], l=2.25[\mathrm{~cm}]$, and $n=0.0[\mathrm{~cm}]$. The voltage transfer ratio $B(x=1.0)$ of the equalizer is calculated from Eq. (15). The result is shown in

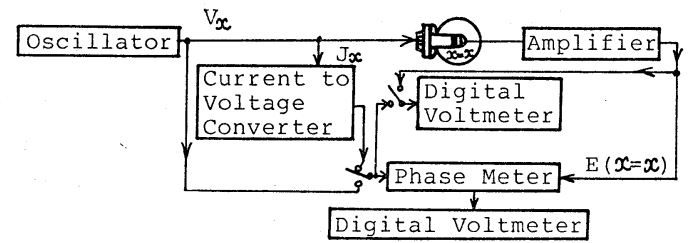

Fig. 14 Experimental arrangement for the measurement of the chain matrix $H$.
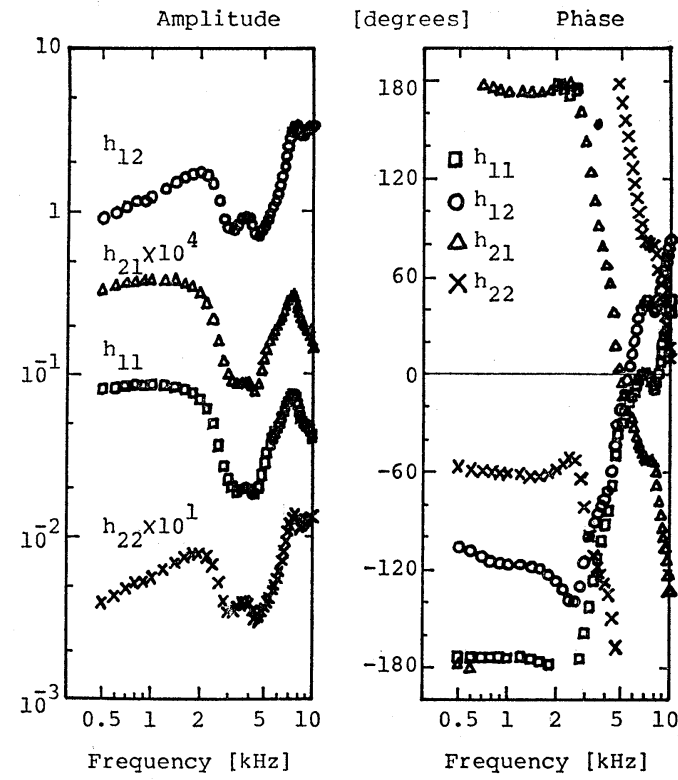

Fig. 15 Elements of the chain matrix $H$ of the headphone attached to the spherical head.

Units are as follows: $h_{11}\left[\mathrm{~V} \cdot \mathrm{cm}^{2} / \mathrm{dyn}\right], h_{12}$ [V $\left.\cdot \mathrm{sec} / \mathrm{cm}^{2}\right], h_{21}\left[\mathrm{~A} \cdot \mathrm{cm}^{3} / \mathrm{dyn}\right]$, and $h_{22}$ [A. $\left.\mathrm{sec} / \mathrm{cm}^{3}\right]$.
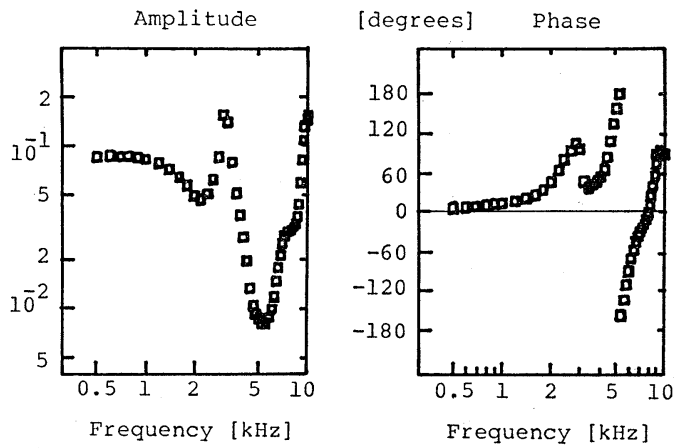

Fig. 16 Voltage transfer ratio $B(x=1.0)$ of the equalizer.

Fig. 16. Here, in the calculation of $B(x=1.0), Z_{\mathrm{s}}$ at $\theta=90^{\circ}$ is substituted.

5.4 Comparison between the Reproduced Sound Pressure $P^{\prime}(x=l)$ and the Original Sound Pressure $P(x=l)$

Figure 17 shows a method of comparing the sound pressure $P^{\prime}(x=l)$ with $P(x=l)$. Here, $P^{\prime}(x=l)$ is computed from the equalizer characteristics 
obtained in Section 5.3 and the chain matrix obtained in Section 5.2.

The comparison between $P^{\prime}(x=l)$ and $P(x=l)$ is done by the comparison between $E^{\prime}(x=l)$ and $E(x=l)$, the following relation being referred to,

$$
\frac{P^{\prime}(x=l)}{P(x=l)}=\frac{A \cdot P^{\prime}(x=l)}{A \cdot P(x=l)}=\frac{E^{\prime}(x=l)}{E(x=l)} .
$$

The comparison is made at four different azimuthal angles of incidence, $\theta=90^{\circ}, 60^{\circ}, 30^{\circ}$, and $0^{\circ}$. For $E(x=m)$ and $E(x=l)$ in Fig. $17, E_{l 1}$ and $E_{l 2}$ obtain-

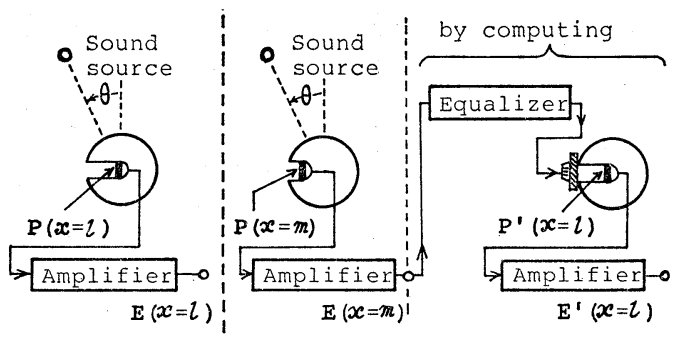

(a) Listener (b) Dummyhead-headphone system

Fig. 17 Method of comparing the reproduced sound pressure in the dummyhead-headphone system $P^{\prime}(x=l)$ with the original sound pressure $P(x=l)$.
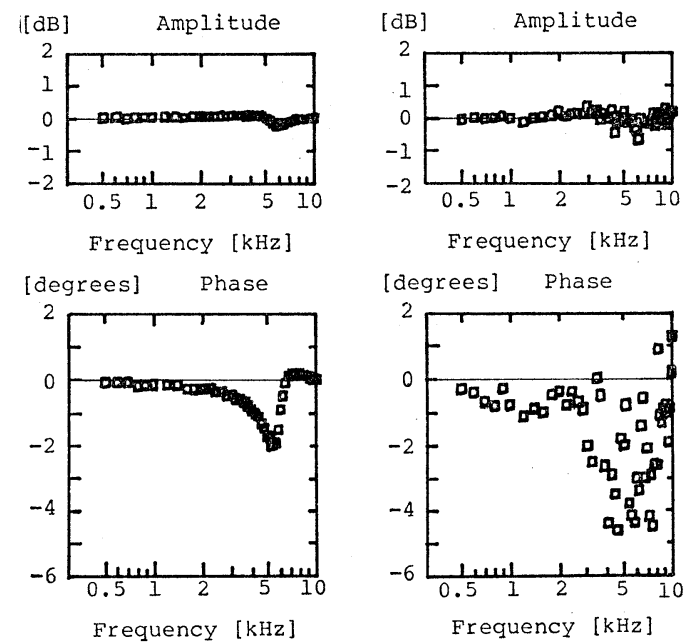

(a)

ed in Section 5.1 are used respectively.

Figure 18 shows the reproduced sound pressure in the dummy-head-headphone system. In the figure, the ratio of $P^{\prime}(x=l)$ to $P(x=l)$ is shown, at two azimuthal angles $\theta=90^{\circ}$ and $0^{\circ}$. Note that the equalizer characteristics used in the computation of $E^{\prime}(x=l)$ is calculated by using $Z_{\mathrm{s}}$ at $\theta=90^{\circ}$.

The followings are observed.

(1) Amplitude ratio of $P^{\prime}(x=l)$ to $P(x=l)$ is within $1 \mathrm{~dB}$.

(2) Phase difference between $P^{\prime}(x=l)$ and $P(x=l)$ is almost within $6^{\circ}$.

\section{CONCLUSION}

The equalization in the dummy-head-headphone system has been discussed in order to enable a listener to hear the same sound as he would hear in the sound field where the dummy-head is located. Deriving the equivalent circuit of the listener in the original sound field and that of the dummy-headheadphone system, we have expressed the necessary characteristics of the equalizer in terms of elements of those equivalent circuits. With the result, the equalizer can be realized on the basis of the human acoustic elements and the headphone. We have also discussed the necessary condition for the characteristics of the equalizer for a specific listener being constant irrespective of the direction of incident sounds and it is found that this requirement for the equalizer is satisfied when the external shape of the dummy-head is identical to that of the listener's head. By the model experiment, the validity of this equalization method has been verified.

In order to realize the equalizer for an actual listener, however, we need more precise knowledge of the dimensions of the earcanal and that of the eardrum impedance over the required frequency range. Furthermore, it remains to be solved what amount of deviation of the shape of a listener's head from that of the dummy-head with its proper equalizer may be allowable.

\section{ACKNOWLEDGEMENTS}

The author would like to express his appreciation to Emertus Prof. Yasuo Makita and Prof. Hikaru Date of Kyushu Institute of Design for their sustained guidance and encouragement, and also to our student, Chikao Muramatu, for his assistance in carrying out experiments. 
K. FUKUDOME: EQUALIZATION FOR THE DUMMY-HEAD-HEADPHONE SYSTEM

\section{REFERENCES}

1) For example, J. J. Zwislocki, "An Acoustic Coupler for Earphone Calibration," Laboratory of Sensory Communication, Syracuse University, New York,
Special Report LSC-S-7, 19-38 (1970).

2) D. P. Egolf and R. G. Leonard, "Experimental Scheme for Analyzing the Dynamic Behavior of Electroacoustic Transducers," J. Acoust. Soc. Am. 62, 1013-1023 (1977). 